\title{
A New Black Hole Solution in Conformal Dilaton Gravity on a Warped Spacetime
}

\author{
Reinoud J. Slagter \\ Asfyon, Astronomisch Fysisch Onderzoek Nederland, Bussum, The Netherlands \\ Email: info@asfyon.com
}

How to cite this paper: Slagter, R.J. (2021) A New Black Hole Solution in Conformal Dilaton Gravity on a Warped Spacetime. Journal of Modern Physics, 12, 1758-1782. https://doi.org/10.4236/jmp.2021.1213103

Received: September 30, 2021

Accepted: November 13, 2021

Published: November 16, 2021

Copyright $\odot 2021$ by author(s) and Scientific Research Publishing Inc. This work is licensed under the Creative Commons Attribution International License (CC BY 4.0).

http://creativecommons.org/licenses/by/4.0/

\begin{abstract}
An exact time-dependent solution of a black hole is found in a conformally invariant gravity model on a warped Randall-Sundrum spacetime, by writing the metric $g_{\mu \nu}=\omega^{\frac{4}{n-2}} \tilde{g}_{\mu \nu}$. Here, $\tilde{g}_{\mu \nu}$ represents the "un-physical" spacetime and $\omega$ the dilaton field, which will be treated on equal footing as any renormalizable scalar field. In the case of a five-dimensional warped spacetime, we thereafter write ${ }^{(4)} \tilde{g}_{\mu \nu}=\bar{\omega}^{2(4)} \bar{g}_{\mu \nu}$. The dilaton field $\bar{\omega}$ can be used to describe the different notion the in-going and outside observers have of the Hawking radiation by using different conformal gauge freedom. The disagreement about the interior of the black hole is explained by the antipodal map of points on the horizon. The free parameters of the solution can be chosen in such a way that $\bar{g}_{\mu \nu}$ is singular-free and topologically regular, even for $\omega \rightarrow 0$. It is remarkable that the 5D and $4 \mathrm{D}$ effective field equations for the metric components and dilaton fields can be written in general dimension $n=4,5$. From the exact energy-momentum tensor in Eddington-Finkelstein coordinates, we are able to determine the gravitational wave contribution in the process of evaporation of the black hole. It is conjectured that, in context of quantization procedures in the vicinity of the horizon, unitarity problems only occur in the bulk at large extra-dimension scale. The subtraction point in an effective theory will be in the UV only in the bulk, because the use of a large extra dimension results in a fundamental Planck scale comparable with the electroweak scale.
\end{abstract}

\section{Keywords}

Conformal Invariance, Dilaton Field, Black Holes, Brane World Models, Antipodal Map

\section{Introduction}

It is believed that the understanding of the quantum mechanical property of a 
black hole is one of the major challenges of modern physics. The quantum features of a black hole were investigated, decades ago, by Hawking in his epic work on radiation effects of a black hole [1] [2]. The thermal emission from the black hole can be described by a temperature $T=\frac{1}{8 \pi M}$, where $M$ is the mass of the black hole. Hawking considered the collapsing body on a background spacetime, which is time dependent and not symmetrical with respect to time reversal.

Vacuum pair-production at the horizon causes the Hawking radiation, which is thermal and contains no information. The anti-particle falls into the black hole. So when the black hole evaporates completely, it seems that information is lost, which is against Quantum Mechanics (QM): it dictates that the initial and final stage of a system is related to a unitary S-matrix. This is a first indication that there is a problem with QM when applied to a black hole spacetime. This is the information paradox.

Related to this issue, is the holographic principle [3], which states that the interior volume of spacetime of a black hole containing the information of the in-going particles is dual to the surface of the horizon. Could it be that the information is still at the horizon? The idea was extended to the well-known Anti-de Sitter/Conformal Field Theory (AdS/CFT) correspondence: in some way, the information must be present in the Hawking radiation. This model relies heavily on string theory, but would solve the information paradox, by introducing the notion of complementarity of the in- and out-side of the black hole. The in-going and out-going particles are entangled and the information of the in-going particle is also reflected back. However, this viewpoint conflicts with causality [4]. The previously emitted Hawking radiation and the corresponding in-going particles are independent systems and at the same time indirect entangled.

Another solution for the information paradox, which doesn't rely on string theory, is the introduction of a firewall [5]. The entanglement between the in-going and out-going particles is broken by a high energetic shield. The freely in-falling observer encounters high-energy particles at the horizon. This viewpoint conflicts with general relativity, i.e., violation of the equivalence principle. Free falling observers, when falling through the horizon, perceive spacetime as Minkowski, so will not notice the horizon at all.

A fundamental issue which is omitted in all the treatments as described above, is the time-dependency of the spacetime structure near the horizon. The emitted Hawking particle will have a back-reaction effect on the spacetime [6] [7]. Could it be possible, that the topology of the black hole must be revised? It is well known that quantum field theory on a curved spacetime opens the possibility that a field theory can have different vacuum states. It can have intrinsic statistical features from a change in topology and not from a priori statistical description of the matter fields.

A spacetime with a given local geometry admits in principle, different possible global topologies. One can consider the modification of the spacetime topology of the form $\hat{\mathcal{M}} / \Gamma$, where $\Gamma$ is a discrete subgroup of isometries of $\mathcal{M}$ [8] 
[9] [10] [11], without fixed points. $\hat{\mathcal{M}}$ is non-singular and is obtained from its universal covering $\mathcal{M}$ by identifying points equivalent under $\Gamma$. A particular interesting case is obtained, when $\Gamma$ is the antipodal transformation on $\mathcal{M}$

$$
J: P(X) \rightarrow \hat{P}(\hat{X}) .
$$

where the light-cone of the antipode of $P(X)$ intersects the light-cone of $P(X)$ only in two point (at the boundary of the spacetime). This is the so-called "elliptic interpretation" [12] of spacetime, where antipodal points represents in fact the same world-point or event. The future and past event horizon intersect each other as a projected cylinder $\mathbb{R}_{1} \times \mathbb{S}_{1} / \mathbb{Z}_{2}{ }^{1}$. At the intersection one then identifies antipodal points. One must realize that the antipodal map is a boundary condition at the horizon, only observable by the outside observer. On a black hole spacetime, the inside is removed. So nothing can escape the interior, since there is no interior. The field theories formulated on $\mathcal{M}$ and $\hat{\mathcal{M}}$ are globally different, while locally $\mathcal{M}$ and $\hat{\mathcal{M}}$ are identically. The emitted radiation is only locally thermal. Antipodal identification, however, destroys the thermal features in the Fock space construction. In the construction, one needs unitary evolution operators for the in-going and out-going particles [13].

In order to avoid wormhole constellations or demanding "an other universe" in the construction of the Penrose diagram, it is essential that the asymptotic domain of $\mathcal{M}$ maps one-to-one onto the ordinary spacetime in order to preserve the metric. In fact, one deals with one black hole. A consequence is that time-inversion takes place in region II of the Penrose diagram, so interchange of the creation and annihilation operators and entangling positive energy particles at the horizon with positive energy antiparticles at the antipodes. So the antipodal identification is not in conflict with the general CPT invariance of our world. Further, for the outside observer, the thermodynamically mixed state is replaced by a pure state. So the Hawking particles at opposite sides of the black hole are entangled.

The former representation that observers have no access to the inside of the black hole is no longer valid. One arrives by this new geometrical description at pure quantum states for the black hole. It will solve, moreover, the information paradox and firewall problem as well ${ }^{2}$.

The gravitational back-reaction as proposed by't Hooft [14] [15], suggests a cut-off of high momenta, which avoids the firewall. The in-going particle has a back-reaction on the other particles, leading to a unitary S-matrix. The gravitational interaction between the in-going and out-going particles will be strong, because we are dealing here with a strongly curved spacetime near the horizon. Using a "cut-and-paste" procedure, one replaces the high-energy particles ("hard"), i.e., mass or momentum of the order of the Planck mass, by low-energy

${ }^{1}$ We work here in polar coordinates, because the spinning black hole we will consider, has a preferred spin axis. The antipodal identification is then $(U, V, z, \varphi) \rightarrow(-U,-V,-z, \pi+\varphi)$.

${ }^{2}$ The technical aspects in constructing the unitary S-matrix can be found in the literature, as provided by the references. 
("soft") particles far away. These hard particles just caused the firewall problem. Hard particles will also influence the local spacetime (to become non-Schwarzschild) and causes the Shapiro effect. The interaction with the soft particles is described by the Shapiro delay. Effectively, all hard particles are quantum clones of all soft particles. By this "firewall-transformation", we look only at the soft particle clones. They define the Hilbert space and leads to a unitary scattering matrix. The net result is that the black hole is actually in a pure state, invalidating the entanglement arguments in the firewall paradox. The entanglement issue can be reformulated by considering the two regions I and II in the maximally extended Penrose diagram of the black hole, as representing two "hemispheres" of the same black hole. It turns out that the antipodal identification keeps the wave functions pure and the central $r=0$ singularity has disappeared. This gravitational deformation will cause transitions from region I to II in the Penrose diagram. The fundamental construction then consists of the exchange of the position operator with the momentum operator of the in-going particles, which turn them into out-particles. Hereby, 't Hooft expands the moment distributions and position variables in partial waves in $(\theta, \varphi)$. So the Hawking particles emerging from I are entangled with the particles emerging from II. An important new aspect is the way particles transmit the information they carry across the horizon. In the new model, the Hawking particles emerging from I are maximally entangled with the particles emerging from II. The particles form a pure state, which solves the information paradox.

In order to describe the more realistic black holes, such as the axially symmetric Kerr black hole, it is not possible to ignore the dynamics of the horizon. Moreover, one must incorporate gravitation waves. There is another reason to consider axially symmetry. A spherical symmetric system cannot emit gravitation waves [16]. Astronomers conjecture that most of the black holes in the center of galaxies are of the Kerr type. A linear approximation is, of course, inadequate in high-curvature situations. In the linear approximation, the waves don't carry enough energy and momentum to affect their own propagation. The notion of the "classical" Hartle-Hawking vacuum thermal state, with a temperature $T \sim \frac{1}{M} \sim \kappa$ and the luminosity $\frac{\mathrm{d} M}{\mathrm{~d} t} \sim-\frac{1}{M^{2}}$ must also be revised when the mass reaches the order of the Planck mass. On the Kerr black hole spacetime no analog of the Hartle-Hawking vacuum state exists. The Killing field $\xi^{\mu}$ generates a bifurcate Killing horizon ( $\xi^{\mu} \xi_{\mu}=-1$ at infinity) and possesses space like orbits near infinity [17].

Another aspect of the huge curvature in the vicinity of the horizon, will be the problem of constructing a renormalizable (and maintaining unitarity) quantum gravity model of the Standard Model fields, which must be incorporated in the Lagrangian. Up till now, no convincing theory of quantum gravity is available. Many attempts were made in order to make a renormalizable and unitary quantum gravity model. One also can try to construct a renormalizable model, by 
adding fourth order derivative terms of the curvature tensor (Euler-term). However, one looses unitarity. Also the "old" effective field theory (EFT) has its problems. One ignores what is going on at high energy. In order to solve the anomalies one encounters in calculating the effective action, one can apply the so-called conformal dilaton gravity (CDG) model [6] [7] [18] [19]. CDG is a promising route to tackle the problems arising in quantum gravity model, such as the loss of unitarity close to the horizon. One assumes local conformal symmetry, which is spontaneously broken (for example by a quartic self-coupling of the Higgs field). Changing the symmetry of the action was also successful in the past, i.e., in the SM of particle physics. A numerical investigation of a black hole solution of a non-vacuum CDG model, was recently performed [20]. The key feature in CDG, is the splitting of the metric tensor $g_{\mu \nu}=\omega^{\frac{4}{n-2}} \tilde{g}_{\mu \nu}$, with $\omega$ the dilaton field. Applying perturbation techniques (and renormalization/ dimensional regularization), in order to find the effective action and its divergencies, one first integrate over $\omega$ (shifted to the complex contour), considered as a conventional renormalizable scalar field and afterwards over $\tilde{g}_{\mu v}$ and matter fields. The dilaton field is locally unobservable. It is fixed when we choose the global spacetime and coordinate system. If one applies this principle to a black hole spacetime, then the energy-momentum tensor of $\omega$ influences the Hawking radiation. When $\tilde{g}_{\mu v}$ is flat, then the handling of the anomalies simplifies considerably [15]. When $\tilde{g}_{\mu v}$ is non-flat, the problems are more deep-seated.

It is well known, that the antipodal transformation, or inversion, is part of the conformal group [21]. So conformal invariant gravity models could fit very well the models of antipodal mapping as described above. In this context, the modification of GRT by an additional spacetime dimension could be an alternative compromise, because Einstein gravity on the brane will be modified by the very embedding itself and opens up a possible new way to address the dark energy problem [22] [23] [24] ${ }^{3}$. These models can be applied to the standard Friedmann-Lemaittre-Robertson-Walker (FLRW) spacetime and the modification on the Friedmann equations can be investigated [25]. Recently, Maldacena, et al. [26], applies the RS model to two black hole spacetimes and could construct a traversable macroscopic wormhole solution by adding only a $5 \mathrm{D} \mathrm{U}(1)$ gauge field (see also Maldacena [27]). However, an empty bulk would be preferable. Instead, one can investigate the contribution of the projected 5D Weyl tensor on the $4 \mathrm{D}$ brane. It carries information of the gravitational field outside the brane. If one writes the 5D Einstein equation in CDG setting, it could be possible that an effective theory can be constructed without an UV cutoff, because the fundamental scale $M_{5}$ can be much less than the effective scale $M_{P l}$ due to the warp factor. The physical scale is therefore not determined by $M_{P l}$.

In this manuscript we will apply the antipodal map on a spinning black hole spacetime in conformal dilaton gravity applied to a warped 5D spacetime.

${ }^{3}$ There is another argument in favor of a (warped) $5 \mathrm{D}$ spacetime. It turns out, as we shall see, that a surface in $4 \mathrm{D}$ can be immersed to $5 \mathrm{D}$, like a Klein bottle. 


\section{Conformal Transformations and Antipodal Mapping}

\subsection{The Origin of the Antipodal Mapping}

The antipodal map originates from the so-called "elliptic" interpretation [12]. If one considers the hyperboloid $\mathrm{H},-t^{2}+x^{2}+y^{2}+z^{2}+w^{2}=R^{2}$, then the spacelike sections through the origin are ellipses and the time-like sections are hyperbola branches.

Since the de Sitter spacetime can be isometrically embedded as a hyperboloid in $\mathbb{R}^{5}$, one can take $R^{2}=-\frac{3}{\Lambda}$. If one suppresses the coordinates $(z, w)$, we have the $\mathbb{R}^{3}$ Minkowski metric. Lorentz transformations (LT's) around the origin transform $\mathrm{H}$ into itself. Circles on $\mathrm{H}$ represent space at different epochs. The bottle-neck parallel is a spatial geodesic, while the others are not. Further, the circumferences contract from $z=-\infty$ to $z=0$ and then expand. A LT of $\mathbb{R}^{3}$ turns the bottle-neck into an ellipse, cut out of $\mathrm{H}$ with an angle $<45^{\circ}$ with the $(x, y)$-plane. See Figure 1 . All the ellipses are equivalent space-like geodesics since each of them is transferred by a suitable automorphism into the bottle-neck, which is one of them. One defines the antipodal map

$$
J: P(t, x, y) \rightarrow \hat{P}(-t,-x,-y),
$$

on $\mathrm{H}$. The antipodicity is Lorentz invariant. When the angle approaches $45^{\circ}$, then the ellipses degenerate into a couple of parallel generators $\left(g_{1}, g_{2}\right)$ (null geodesics). The other plane of $45^{\circ}$ delivers the set $\left(g_{3}, g_{4}\right)$. The sets $\left(g_{1}, g_{4}\right)$ and $\left(g_{2}, g_{3}\right)$ form, for example, the light-cones at the points $M$ and $\hat{M}$. If one
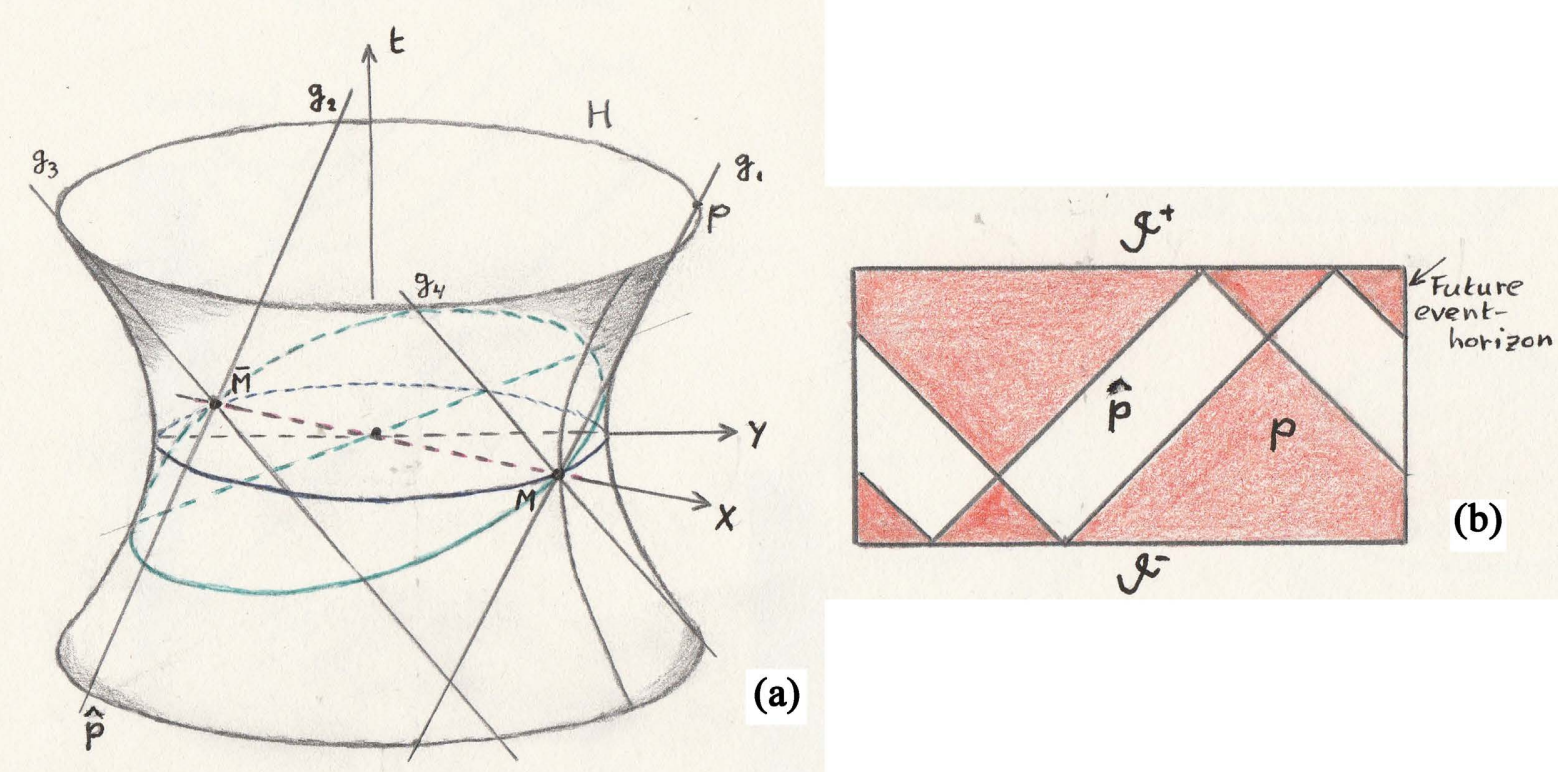

(a)

Figure 1. Hyperboloid $H$ representing the $\mathbb{R}^{3}$ spacetime of the compactified de Sitter universe $\mathbb{R}^{5}((z, w)$ suppressed) (a). In the Penrose diagram, the antipodal points $P$ and $J(P)$ are spacelike separated. An observer moving in de Sitter spacetime cannot meet both $P$ and $J(P)$ (b). He cannot receive a message from $P, J(P)$. Moreover, he cannot receive a message from $P$ and send a message to $J(P)$. 
moves upwards along t, the inner angles of the light-cones decrease. Note that the light-cones at $P$ and $\hat{P}$ has no point in common and the antipodes are joined by a space-like geodesic. Now Schrödinger proposed to identify $P$ and $\hat{P}$ with the same physical world-point or event. One half of $\mathrm{H}$, containing no antipodal points, represents the "whole world". Thereafter, Schrödinger argues in a clever way that the total potential of experiences of any observer is complete and embraces the same events for any two observers, whatever their world lines be. But there is a price we have to pay for ${ }^{4}$. The direction of the arrow of time is lost (or the distinction between the "fore-cone" and "after-cone" is lost). The allotment of past and future is undecidable. The elliptic model is time-reversible. This can open perspective to the general CPT invariance of our world. The real problems arise, when one considers thermodynamical systems, as is the case for the Hawking effect in the vicinity of the horizon of a black hole. Then the entropy comes into play. Note, quoting Schrödinger, "the irreversible laws of thermodynamics can only be based on the statistical microscopically reversible systems on condition that statistical theory be autonomous in defining the arrow of time. If any other law of nature determines this arrow, the statistical theory collapses."

In a pseudo-polar frame $(\chi, T, \theta, \varphi)$ we can write the line element

$$
\mathrm{d} s^{2}=-R^{2} \mathrm{~d} T^{2}+R^{2} \cosh ^{2} T\left[\mathrm{~d} \chi^{2}+\sin ^{2} \chi\left(\mathrm{d} \theta^{2}+\sin ^{2} \theta \mathrm{d} \varphi^{2}\right)\right],
$$

where $0<\chi<2 \pi$. The antipodal map becomes now

$$
J:(T, \chi, \theta, \varphi) \rightarrow(-T, \pi+\chi, \pi-\theta, \pi+\varphi) .
$$

We already mentioned that de Sitter can be embedded as a hyperboloid in $5 \mathrm{D}$ Minkowski. We then say that $J: X^{\mu} \rightarrow-X^{\mu}$ is an inversion ${ }^{5}$. There exist another coordinate system (introduced by de Sitter himself) in which the line element is written as

$$
\mathrm{d} s^{2}=-\left(1-\frac{\rho^{2}}{R^{2}}\right) \mathrm{d} T^{\prime 2}+\frac{1}{1-\frac{\rho^{2}}{R^{2}}} \mathrm{~d} \rho^{2}+\rho^{2}\left(\mathrm{~d} \theta^{\prime 2}+\sin ^{2} \theta^{\prime} \mathrm{d} \varphi^{\prime 2}\right),
$$

where we have taken the velocity of the LT $\tanh T=\frac{t}{y}$. This is the static de Sitter and the spaces of constant time are all equivalent. There are singularities for $x= \pm R \quad\left(\chi= \pm 90^{\circ}\right)$, i.e., the points $(M, \hat{M})$. However, as also observed by Schrödinger, this static model is not adequate for applying the antipodal map. In order to apply the antipodal map on a black hole spacetime in a more general setting, one needs a time dependent spacetime.

${ }^{4}$ This price is worth paying in the black hole situation, when the information paradox will be solved by the antipodal map. The antipodal half is not time orientable. There is a breakdown of the global distinction between past and future in the interior of the black hole.

${ }^{5}$ The inversion $X_{\mu} \rightarrow-\frac{X_{\mu}}{X^{2}}$ (as well as the dilatations) is part of the conformal group [21]. We shall see in the next sections that in general the conformal group is a projective group from $5 \mathrm{D}$. The fifth "degree of freedom" is a sort of gauge space. 


\subsection{The "Classical" Hawking Effect and Its Problems}

The famous result of Hawking states, that a black hole will radiate at "sufficiently" late times like a black body at a temperature

$$
k T \sim \frac{\kappa}{2 \pi}=\frac{\hbar c^{3}}{8 \pi G M},
$$

with $\kappa$ the surface gravity and $M$ the mass. The entropy should then be $S_{b h}=\frac{k c^{3}}{4 \hbar} A$, with $A$ the area of the horizon. However, one runs into problems by the back-reaction effect of the particle creation, which will alter the area. It is questionable if the ordinary laws of thermodynamics can be applied to a black hole. It is clear that these laws must be constrained to form quantum states with orthonormality and unitarity conditions. Suppose that an isolated black hole completely evaporate within a finite time. Loss of quantum coherence should then occur i.e., an initially pure quantum state should evolve to a mixed state. In general, in the classical picture, a black hole cannot causally influence its exterior, so it is hard to understand the mechanism by which thermal equilibrium could be achieved. Observe that the state of the field at late times in the region I of the Penrose diagram (and so the particles flux reaching infinity) is described by a density matrix by the S-matrix analysis. The particles present in region I are strongly correlated with the particles which entered the black hole at earlier times.

Consider now in Figure 2 the evolution of two Cauchy surfaces ("time" $\Sigma_{1}$
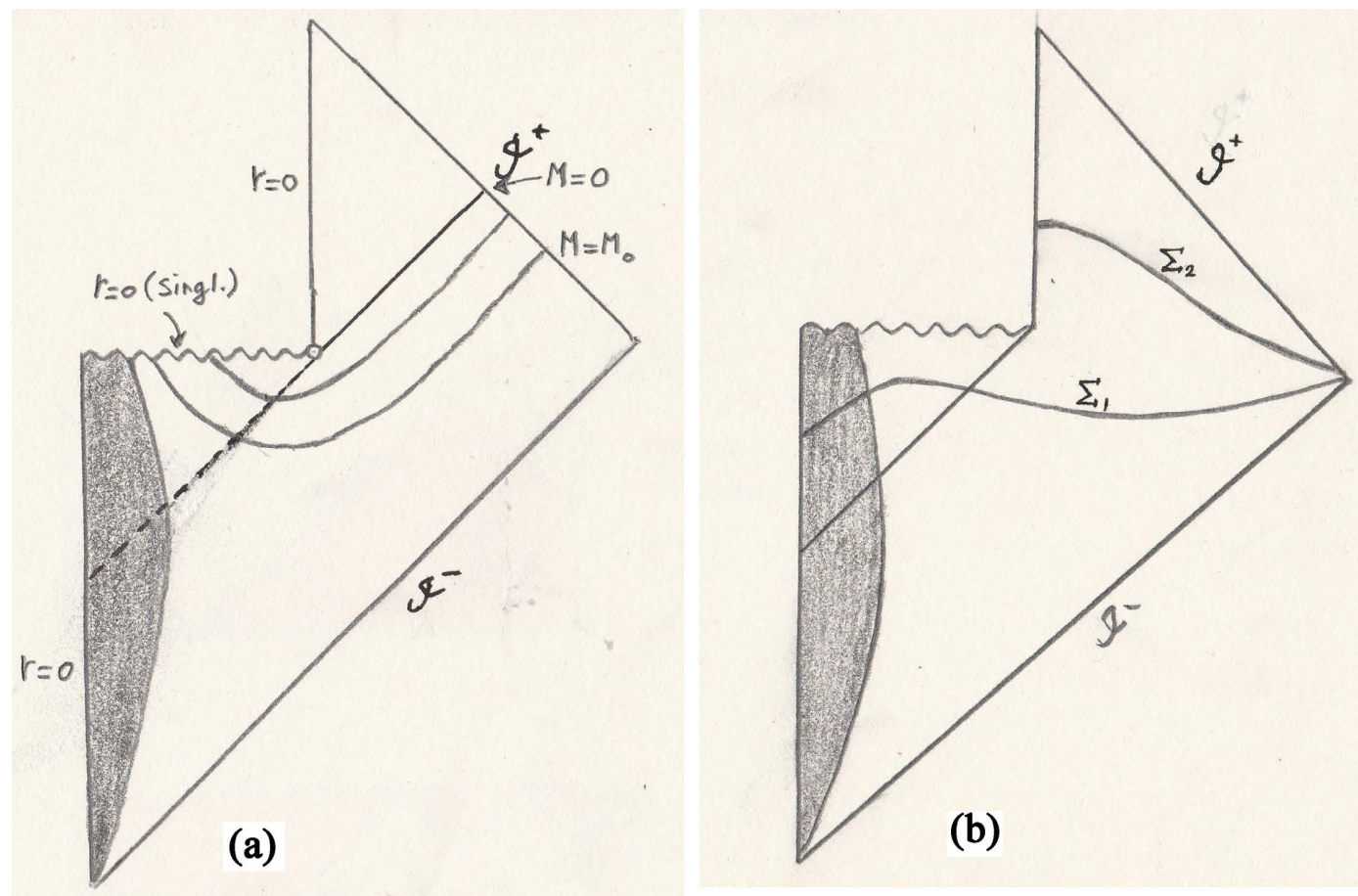

Figure 2. The formation and evaporation of the Schwarschild black hole. The contour $M=0$ lies at the retarded time corresponding to the final evaporation (a). The geometry is flat above this contour. It turns out that there will be loss of quantum coherence, i.e., an evolution from a pure state to a mixed state [17], as can be observed by the two Cauchy surfaces $\Sigma_{1}$ and $\Sigma_{2}$ (b). 
to "time" $\Sigma_{2}$ ). When the black hole disappears from the spacetime, then at late times, the entire state of the field is mixed. If one takes the "out" Hilbert space to be the Fock space of the particles propagating out to infinity at late times, one cannot describe particle creation and scattering by an ordinary S-matrix. The initial pure state will evolve to a final density matrix. So we have a breakdown of quantum theory. The antipodal model, however, could "repair" this breakdown.

\subsection{Conformal Map between Manifolds and the Antipodal Map}

Let us consider a smooth regular map $f: M^{n} \curvearrowright N^{n}$, with metrics $g_{1}$ and $g_{2}$ [21]. This represents a local isometry if it preserves the metric, i.e., $f * g_{2}=g_{1}$ and a global isometry if it is a diffeomorphism too. It is a conformal map if it rescales the metric, i.e., $f * g_{2}=\Omega^{2}(x) g_{1}$, with $\Omega^{2}$ a positive scalar field. Moreover, it preserves the light-cone structure. Further, an isometry maps geodesics into geodesics and preserves the affine parameter. Conformal maps preserve null geodesics. In many physical applications, it is preferable to consider global isometries: they constitute a group of the manifold. On Minkowski spacetime, the diffeomorphism is of the form $y^{\alpha}=A_{\beta}^{\alpha}+b^{\alpha}$, with $A_{\beta}^{\alpha}$ a Lorentz matrix. In this context, one must not confuse this transformation with the Poincare transformations, which are of the same form. They connect two inertial frames. They are the basic of special relativity. They are coordinate transformations and are linear. Conformal maps in Minkowski spacetime do not act as linear transformations. Nevertheless, one can generate them from linear transformations in a higher-dimensional spacetime. Now the antipodal map can be represented as a conformal transformation generated from pseude-othogonal matrices of $O(3)$, i.e., the conformal group. Each conformal transformation in this group can be presented by a pair of antipodal matrices. In language of group theory, the map of a pair of antipodal points into a pair of antipodal points can be considered as a conformal transformation on $M\left(\mathbf{R}^{1} \otimes \mathbf{R}^{1}\right)$ and is represented by the pseudo-orthogonal group of matrices $O(2,2)$. The matrix $-\mathbb{I}$ will interchange antipodal points. Details can be found in Felsager [21]. A very illuminating presentation of conformal transformations, in particular the inversions, can be given by the stereographic projection $\left(S P: S^{2} \rightarrow \mathbb{C}_{\infty}\right)$ by using complex numbers $z \in \mathbb{C}$. If one extend the complex plane, $\mathbb{C}_{\infty}=\mathbb{C} \cup \infty$, then one has a bijection between $\mathbb{C}_{\infty}$ and $S^{2}$. This is the Riemann sphere and one says that $\mathbb{C}_{\infty}$ is a one-point compactification. Moreover, the map is a homeomorphism. Further, $S P^{-1}$ are conformal maps. The inversion map $T(z)=\frac{1}{z}=\frac{\bar{z}}{|z|^{2}}$ is a conformal map in $\mathbb{C}_{\infty} \rightarrow \mathbb{C}_{\infty}$. One can proof that the Möbius transformations $M(\mathbb{C})$ $f: \mathbb{C}_{\infty} \rightarrow \mathbb{C}_{\infty}$ with $f(z)=a z+b / c z+b$, are the conformal maps of $\mathbb{C}_{\infty}$. The set $M(\mathbb{C})$ is a surjective group with a homomorphism $\Gamma: G L_{2}(\mathbb{C}) \rightarrow M\left(\mathbb{C}_{\infty}\right)$ and kernel the diagonal matrices. The group $G L_{2}(\mathbb{C}) / k I$ is the $P G L_{2}(\mathbb{C})_{\infty}$, with $k$ a constant. If $S L_{2}(\mathbb{C})$ represents the complex matices with determinant 1 , then $\Gamma: S L_{2}(\mathbb{C}) \rightarrow M\left(\mathbb{C}_{\infty}\right)$ is onto and has kernel $\pm I$. One then has an 
isomorphism $\Gamma: S L_{2}(\mathbb{C}) / \pm I \rightarrow M\left(\mathbb{C}_{\infty}\right)$. The class of the Möbius transformations where $a, b, c, d$ are $\in \mathbb{R}$, are interesting, because they apply to hyperbolic geometry. The group $\operatorname{PSL}_{2}(\mathbb{C})$ can then be defined, in order to define conjugate classes and to classify the fixed points, that means in our situation, no fixed points. If an element $f \in(\mathbb{C})$ has period $\mathrm{m}$ with $f^{M}(z)=z$ for the smallest $m$, then $f$ has no fixed points. Rotations in $(\mathbb{C})_{\infty}$ are also Möbius transformations. A rotation of $S^{2}$ is a linear map with positive determinant that maps $S^{2}$ onto itself. Because there is a fixed axis, one can represent the rotation in $\mathbb{R}^{3}$ (the $S O(3)$, the orthogonal matrices with determinant 1) by

$$
A=\left(\begin{array}{ccc}
\cos \theta & \sin \theta & 0 \\
-\sin \theta & \cos \theta & 0 \\
0 & 0 & 1
\end{array}\right)
$$

They are conformal maps of $\mathbb{R}^{3}$. A map $f: \mathbb{C} \rightarrow \mathbb{C}$ is a rotation in $\mathbb{C}$ if $S P^{-1} \circ f \circ S P: S^{2} \rightarrow S^{2}$. So $f$ is conformal too. Suppose $P=(u, v, w) \in S^{2}$, and $\bar{P}=(-u,-v,-w) \in S^{2}$ is the antipodal point in $S^{2}$. Then, if $z=S P((u, v, w)) \in \mathbb{C}_{\infty}$, the antipodal point of $z, \bar{z} \in \mathbb{C}_{\infty}$ is given by $\frac{-1}{\bar{z}}$. So if $f \in \operatorname{Rot}\left(\mathbb{C}_{\infty}\right)$, then the antipodal pair $(z, \bar{z})$ is mapped to an antipodal pair $(f(z), f(\bar{z}))$. Further, one proofs that $f\left(\frac{-1}{\bar{z}}\right)=\frac{-1}{\overline{f(z)}}$ and $\operatorname{Rot}\left(\mathbb{C}_{\infty}\right)=\operatorname{PSU}_{2}(\mathbb{C})$ is isomorphic with $S O(3)$. So $S O(3)$ will generate the conformal group. which can be used in our $4 \mathrm{D}$ spacetime, specially the inversion (by defining self-dual and anti-self-dual forms). One then can formulate the Cauchy-Riemann equations. In physics, they play an important role, because the solution of these equations is automatically a harmonic function of the Laplace equation. Moreover, the equations are conformally invariant.

There is another interesting application of the Möbius presentation: define a complex manifold in 4D. This is the Ernst formulation [28]. If one introduces two complex metric components, one reformulates the Kerr spacetime in a very transparent way. Non-vacuum models can then be generated from the vacuum situation. Just as the holomorphic smooth mappings on the complex manifold of the Riemann sphere $f: S^{2} \rightarrow S^{2}$. These mapping are conformal if they are holomorphic. It could be well possible to extend this approach to $4 \mathrm{D}$. A holomorphic map has interesting properties. It can be represented by an algebraic function $f(z)=P(z) / Q(z)$, with $(P, Q)$ polynomials. So smooth function can be replaced by a holomorphic one. Further, the polynomials can have zero's or singular points, real or complex. Compare this with the conformal maps on the Riemann sphere (generated by the inverse stereographic projection), where the north and south poles causes poles. Some notes must be made about the antipodal map when one uses polar coordinates $(\theta, \varphi)$ on $S^{2}$ of $\left(\theta_{0}, \varphi\right) \rightarrow\left(\theta_{0}, n \varphi\right)$ (rotation over the azimuthal angle $n \varphi$, with $n$ the winding number). It is singular at the poles, unless we take $\cos ^{2} n \varphi+\sin ^{2} n \varphi=1$, which is true for $n= \pm 1$. For 
$n=-1$ we have the antipodal map!

Remember, when adding a scalar gauge field to the Lagrangian (which becomes the axially symmetric Nielsen-Olesen vortex), $\mathrm{n}$ represents the number of magnetic flux quanta. It is conjectured that the antipodal map can be applied to our exact solution presented in the next sections.

\section{The Black Hole Solution on a 5D Warped Spacetime in Conformal Dilaton Gravity}

\subsection{The 5D Warped Spacetime}

Instead of considering the static metric of de Sitter, i.e., Equation (5), we will now investigate the dynamical 5D spacetime warped spacetime [23] [24] [25]

$\mathrm{d} s^{2}=\omega(t, r, y)^{2}\left[-N(t, r)^{2} \mathrm{~d} t^{2}+\frac{1}{N(t, r)^{2}} \mathrm{~d} r^{2}+\mathrm{d} z^{2}+r^{2}\left(\mathrm{~d} \varphi+N^{\varphi}(t, r) \mathrm{d} t\right)^{2}+\mathrm{d} y^{2}\right],(7)$

where $y$ is the extra dimension and $\omega$ a warp factor in the formulation of Randall-Sundrum's (RS) 5D warped spacetime with one large extra dimension and negative bulk tension $\Lambda_{5}$. The Standard Model (SM) fields are confined to the $4 \mathrm{D}$ brane, while gravity acts also in the fifth dimension. Originally, the RS model was applied to a 5-dimensional anti-de Sitter (AdS) spacetime with a positive brane tension. This is the so-called RS- 1 model, with one brane. The RS- 2 model treats two branes with $\mathbb{Z}_{2}$ symmetry. However, the effective cosmological constant on the brane can be zero by fine tuning with the negative $\Lambda_{5}$. In the RS model there is a bound state of the graviton confined to the wall as well as a continuum of Kaluza-Klein (KK) states. Four dimensional gravity is then recovered on the brane and the hierarchy problem seems to be solved. Since the pioneering publication of RS, many investigation were done in diverge domains. In particular, Shiromizu et al. [30], extended the RS model to a fully covariant curvature formalism. See also the work of Maartens [31]. It this extended model, an effective Einstein equation is found on the brane, with on the right-hand side a contribution from the $5 \mathrm{D}$ Weyl tensor which carries information of the gravitational field outside the brane. So the brane world observer may be subject to influences from the bulk. The field equations are (were we took an empty bulk) [20]

$$
\begin{gathered}
{ }^{(5)} G_{\mu v}=-\Lambda_{5}{ }^{(5)} g_{\mu \nu}, \\
{ }^{(4)} G_{\mu v}=-\Lambda_{e f f}{ }^{(4)} g_{\mu v}+\kappa_{4}^{2(4)} T_{\mu v}+\kappa_{5}^{4} \mathcal{S}_{\mu \nu}-\mathcal{E}_{\mu \nu},
\end{gathered}
$$

where we have written

$$
{ }^{(5)} g_{\mu v}={ }^{(4)} g_{\mu v}+n_{\mu} n_{v},
$$

with $n^{\mu}$ the unit normal to the brane. Here ${ }^{(4)} T_{\mu \nu}$ is the energy-momentum tensor on the brane and $S_{\mu v}$ the quadratic contribution of the energy-momentum tensor ${ }^{(4)} T_{\mu v}$ arising from the extrinsic curvature terms in the projected Eins- 
tein tensor. Further,

$$
\mathcal{E}_{\mu v}={ }^{(5)} C_{\beta \rho \sigma}^{\alpha} n_{\alpha} n^{\rho(4)} g_{\mu}^{\beta(4)} g_{v}^{\sigma},
$$

represents the projection of the bulk Weyl tensor orthogonal to $n^{\mu}$. The effective gravitational field equations on the brane are not closed. One must solve at the same time the $5 \mathrm{D}$ gravitation field in the bulk.

\subsection{The Conformal Dilaton Gravity (CDG) Model on a 5D Warped Spacetime}

One can distinguish several possible "routes" to the unification of GR and QFT. One can start, for example, with a given classical theory and applies heuristic quantization rules. One then can make a division in canonical and covariant approaches, i.e., uses a Hamiltonial formalism or employs covariance at some stage. The CDG model we consider here, is part of the covariant approach to quantum gravity. The key feature in CDG, is the splitting of the metric tensor

$$
g_{\mu v}=\omega^{\frac{4}{n-2}} \tilde{g}_{\mu v},
$$

with $\omega$ the dilaton field and $\tilde{g}_{\mu v}$ the "“un-physical” spacetime. At high energy, $\omega$ will be treated as a (renormalizable) quantum field. One can prove that the action (without matter terms for the time being)

$$
S=\int \mathrm{d}^{n} x \sqrt{-\tilde{g}}\left[\frac{1}{2} \xi \omega^{2} \tilde{R}+\frac{1}{2} \tilde{g}^{\mu v} \partial_{\mu} \omega \partial_{\nu} \omega+\Lambda \kappa^{\frac{4}{n-2}} \xi^{\frac{n}{n-2}} \omega^{\frac{2 n}{n-2}}\right],
$$

is conformal invariant under

$$
\tilde{g}_{\mu v} \rightarrow \Omega^{\frac{4}{n-2}} \tilde{g}_{\mu v}, \quad \omega \rightarrow \Omega^{-\frac{n-2}{2}} \omega .
$$

The covariant derivative is taken with respect to $\tilde{g}_{\mu v}$. For details, see Slagter [20]. Now we implement the 5D warped spacetime Equation (7). So

$$
{ }^{(5)} g_{\mu v}=\omega^{4 / 3(5)} \tilde{g}_{\mu v}, \quad{ }^{(5)} \tilde{g}_{\mu v}={ }^{(4)} \tilde{g}_{\mu v}+n_{\mu} n_{v},
$$

and write again

$$
{ }^{(4)} \tilde{g}_{\mu v}=\bar{\omega}^{2} \bar{g}_{\mu v} \text {. }
$$

Variation of the action leads to the field equations

$$
\xi \omega \tilde{R}-\tilde{g}^{\mu \nu} \tilde{\nabla}_{\mu} \tilde{\nabla}_{v} \omega-\frac{2 n}{n-2} \Lambda \kappa^{\frac{4}{n-2}} \frac{n}{\xi^{n-2}} \omega^{\frac{n+2}{n-2}}=0
$$

and

$$
\omega^{2} \tilde{G}_{\mu \nu}=T_{\mu \nu}^{\omega}-\Lambda \tilde{g}_{\mu \nu} \kappa^{\frac{4}{n-2}} \xi^{\frac{2}{n-2}} \omega^{\frac{2 n}{n-2}},
$$

with

$$
T_{\mu \nu}^{\omega}=\tilde{\nabla}_{\mu} \tilde{\nabla}_{v} \omega^{2}-\tilde{g}_{\mu \nu} \tilde{\nabla}^{2} \omega^{2}+\frac{1}{\xi}\left(\frac{1}{2} \tilde{g}_{\alpha \beta} \tilde{g}_{\mu \nu}-\tilde{g}_{\mu \alpha} \tilde{g}_{\nu \beta}\right) \partial^{\alpha} \omega \partial^{\beta} \omega .
$$

From the 5D Einstein equations Equation (8) one obtains 
$\omega(t, r, y)=\omega_{1}(t, r) \omega_{2}(y)$, with $\omega_{2}(y)=l=$ constant (the length scale of the extra dimension). The dilaton equations Equation (17) is superfluous. Note that the effective Einstein equations Equation (9) contains the $\mathcal{E}_{\mu v}$, while $T_{\mu v}$ and $\mathcal{S}_{\mu v}$ are taken zero in our case. The dilaton equation is again superfluous.

It turns out that one can write the field equations for $\omega$ and $N$ in the form $(n=4,5)$

$$
\begin{gathered}
\ddot{\omega}=-N^{4} \omega^{\prime \prime}+\frac{n}{\omega(n-2)}\left(N^{4} \omega^{\prime 2}+\dot{\omega}^{2}\right), \\
\ddot{N}=\frac{3 \dot{N}^{2}}{N}-N^{4}\left(N^{\prime \prime}+\frac{3 N^{\prime}}{r}+\frac{N^{\prime 2}}{N}\right) \\
-\frac{n-1}{(n-3) \omega}\left[N^{5}\left(\omega^{\prime \prime}+\frac{\omega^{\prime}}{r}+\frac{n}{2-n} \frac{\omega^{\prime 2}}{\omega}\right)+N^{4} \omega^{\prime} N^{\prime}+\dot{\omega} \dot{N}\right] .
\end{gathered}
$$

One can solve these equations exact (we took $\Lambda_{\text {eff }}=0$ ):

$$
\omega=\left(\frac{a_{1}}{\left(r+a_{2}\right) t+a_{3} r+a_{4}}\right)^{\frac{1}{2} n-1}, N^{2}=\frac{1}{5 r^{2}} \frac{10 a_{2}^{3} r^{2}+20 a_{2}^{2} r^{3}+15 a_{2} r^{4}+4 r^{5}+C_{1}}{C_{2}\left(a_{3}+t\right)^{4}+C_{3}},
$$

with $a_{i}$ some constants. There is a constraint equation

$$
\bar{\omega}^{\prime \prime}=-\frac{2 n}{n-2} \frac{\Lambda l \kappa^{\frac{4}{(n-2}} \xi^{\frac{n-2}{4(n-1)}} \frac{n+2}{\bar{\omega}^{n-2}}}{N^{2}}-\frac{\omega^{\prime} N^{\prime}}{N}-\frac{\omega^{\prime}}{2 r}+\frac{4}{n-2} \frac{\dot{\bar{\omega}}^{2}}{\bar{\omega} N^{4}}-\frac{\dot{\bar{\omega}} \dot{N}}{N^{5}},
$$

which $l$ the dimension of $y$. The solution for the two dilaton fields $\omega$ and $\bar{\omega}$ differs only by the different exponent $\frac{3}{2}$ and 1 respectively. The solution for the metric component is the same (apart from the constants). The solution for the angular momentum component is

$$
N^{\varphi}=F_{n}(t)+\int \frac{1}{r^{3} \bar{\omega}^{\frac{n-1}{n-3}}} \mathrm{~d} r .
$$

The Ricci scalar for $\bar{g}_{\mu v}(\Lambda=0)$ is given by

$$
\bar{R}=\frac{12}{N^{2}}\left[\dot{\bar{\omega}}^{2}-N^{4} \bar{\omega}^{2}\right],
$$

with is consistent with the null condition for the two-dimensional $(t, r)$ line element, when $\bar{R}=0$. One can easily check that the trace of the Einstein equations is zero. Note that $N^{2}$ can be written as

$$
N^{2}=\frac{4 \int r\left(r+a_{2}\right)^{3} \mathrm{~d} r}{r^{2}\left[C_{2}\left(a_{3}+t\right)^{4}+C_{3}\right]} .
$$

So the spacetime seems to have two poles. However, the $r=0$ is questionable. The conservation equations become

$$
\bar{\nabla}^{\mu} \mathcal{E}_{\mu \nu}=\bar{\nabla}^{\mu}\left[\frac{1}{\bar{\omega}^{2}}\left(-\Lambda \kappa^{2\left({ }^{(4)}\right.} \bar{g}_{\mu \nu} \bar{\omega}^{4}+{ }^{(4)} T_{\mu \nu}^{(\bar{\omega})}\right)\right],
$$

which yields differential equations for $\ddot{N}^{\prime}$ and $\dot{N}$ as boundary conditions at 
the brane. It can be described as the non-local conservation equation. In the high energy case close to the horizon, one must include the $\mathcal{S}_{\mu v}$ term. So the divergence of $\mathcal{E}_{\mu \nu}$ is constrained. In the non-conformal case, Equation (27) contains on the right hand side also the quadratic correction $\mathcal{S}_{\mu v}$ of the matter fields on the brane. The effective field equations, Equation (9), are then not a closed system. One needs the Bianchi equations. In fact, $\mathcal{E}_{\mu v}$ encodes corrections from the $5 \mathrm{D}$ graviton effects and are for the brane observer non-local. In our model under consideration, we have only the $T_{\mu \nu}^{(\omega)}$ term and no source terms (only the 5D $\Lambda_{5}$ ). But it still sources the KK modes. The dilaton $\omega$ plays the role of a "scalar field". But we don't need the 5D equations themselves, because the solution for $N$ is the same! It is only the $\omega^{4 / 3}$ which represents the $5 \mathrm{D}$ contribution. There is no exchange of energy-momentum between the bulk and brane. If one applies the model to a FLRW model [31], then the evolution equations are very complicated. Inhomogeneous and anisotropic effects from the $4 \mathrm{D}$ matter radiation distribution on the brane are sources for the $5 \mathrm{D}$ Weyl tensor $\mathcal{E}_{\mu \nu}$ and cause non-local back-reaction on the brane. One needs an approximation scheme in order to find the missing evolution equation for $\mathcal{E}_{\mu \nu}$.

The locations of the horizon's and ergo-spheres are found by solving $N^{2}=0$ and $\bar{g}_{t t}=0$ respectively. $N^{2}$ becomes singular at coordinate time $t=t_{H}=-b_{3}+\sqrt[4]{-\frac{C_{3}}{C_{2}}}$. However, $\bar{g}_{\mu v}$ can be made regular everywhere and singular free by suitable choices of the parameters $b_{i}, c_{i}$ and $C_{i}$. For $C_{1}=0, \bar{g}_{\mu \nu}$ has one real zero $r_{H}=\sim\left|1.606 b_{2}\right|$ and two complex zero's $\sim(0.178 \pm 0.638 I) b_{2}$. In Figure 3, we plotted the possible graphs. If one ignores the contribution from the bulk, then $N^{2}$ has for $C_{1}=0$ no real roots, so only naked singularities. The contribution from the bulk then generates at least one horizon.

\subsection{Penrose Diagram}

If we define the coordinates, $\mathrm{d} r^{*} \equiv \frac{1}{N_{1}(r)^{2}} \mathrm{~d} r$ and $\mathrm{d} t^{*} \equiv N_{2}(t)^{2} \mathrm{~d} t$, then our induced spacetime can be written as

$$
\mathrm{d} s^{2}=\omega^{4 / 3} \bar{\omega}^{2}\left[\frac{N_{1}^{2}}{N_{2}^{2}}\left(-\mathrm{d} t^{* 2}+\mathrm{d} r^{* 2}\right)+\mathrm{d} z^{2}+r^{2}\left(\mathrm{~d} \varphi+\frac{N^{\varphi}}{N_{2}^{2}} \mathrm{~d} t^{*}\right)^{2}\right],
$$

with

$$
N_{1}^{2}=\frac{10 b_{2}^{3} r^{2}+20 b_{2}^{2} r^{3}+15 b_{2} r^{4}+4 r^{5}+C_{1}}{5 r^{2}}, N_{2}^{2}=\frac{1}{C_{2}\left(t+b_{3}\right)^{4}+C_{3}}
$$

and

$$
r^{*}=\frac{1}{4} \sum_{r_{i}^{H}} \frac{r_{i}^{H} \log \left(r-r_{i}^{H}\right)}{\left(r_{i}^{H}+b_{2}\right)^{3}}, \quad t^{*}=\frac{1}{4 C_{2}} \sum_{t_{i}^{H}} \frac{\log \left(t-t_{i}^{H}\right)}{\left(t_{i}^{H}+b_{3}\right)^{3}} .
$$

The sum it taken over the roots of $\left(10 b_{2}^{3} r^{2}+20 b_{2}^{2} r^{3}+15 b_{2} r^{4}+4 r^{5}+C_{1}\right)$ and 

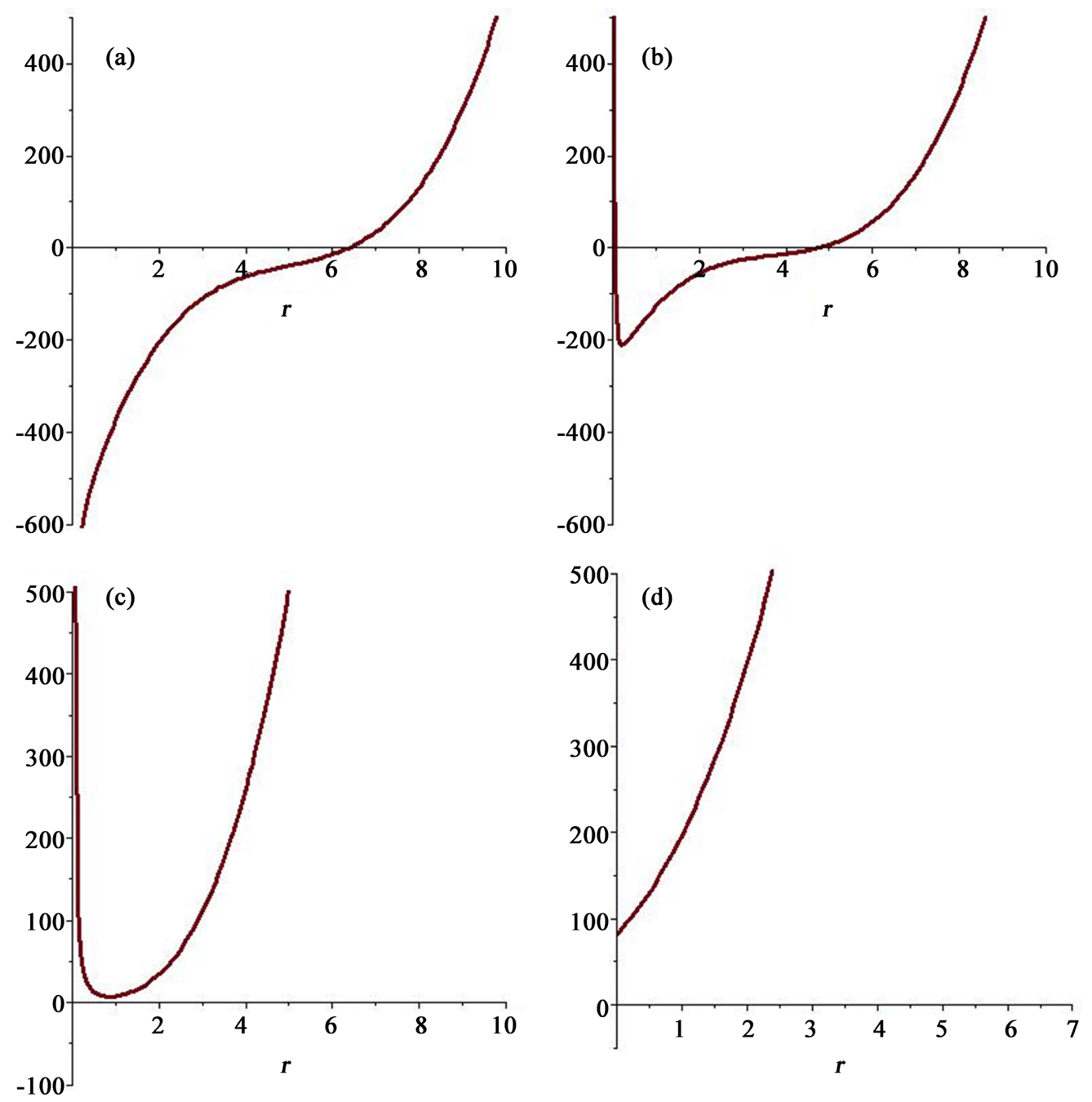

Figure 3. Four possible plots of $N^{2}$ as function of $r$.

$C_{2}\left(t+b_{3}\right)^{4}+C_{3}$, i.e., $r_{i}^{H}$ and $t_{i}^{H}$. This polynomial in $r$ defining the roots of $N_{1}^{2}$, is a quintic equation, which has some interesting connection with Klein's icosahedral solution (see appendix). Further, one can define the azimuthal angular coordinate $\mathrm{d} \varphi^{*} \equiv\left(\mathrm{d} \varphi+\frac{N^{\varphi}}{N_{2}^{2}} \mathrm{~d} t^{*}\right)$, which can be used when an incoming null geodesic falls into the event horizon. $\varphi^{*}$ is the azimuthal angle in a coordinate system rotating about the $\mathrm{z}$-axis relative to the Boyer-Lindquist coordinates. Next, we define the coordinates [32] (in the case of $C_{1}=C_{3}=0$ and 1 horizon, for the time being)

$$
\begin{aligned}
& U_{+}=\mathrm{e}^{\kappa\left(r^{*}-t^{*}\right)}, \quad V_{+}=\mathrm{e}^{\kappa\left(r^{*}+t^{*}\right)} \quad r>r_{H} \\
& U_{-}=-\mathrm{e}^{\kappa\left(r^{*}-t^{*}\right)}, \quad V_{-}=-\mathrm{e}^{\kappa\left(r^{*}+t^{*}\right)} \quad r<r_{H},
\end{aligned}
$$

with $\kappa$ a constant. The spacetime becomes

$$
\mathrm{d} s^{2}=\omega^{4 / 3} \bar{\omega}^{2}\left[\frac{N_{1}^{2}}{N_{2}^{2}} \log (U V)^{\frac{1}{2 \kappa}} \mathrm{d} U \mathrm{~d} V+\mathrm{d} z^{2}+r^{2} \mathrm{~d} \varphi^{* 2}\right] .
$$


In Figure 4, we plotted the Penrose diagram (a). The antipodal points $P(X)$ and $\bar{P}(\bar{X})$ are physically identified. If we compactify the coordinates,

$$
\tilde{U}=\tanh U, \quad \tilde{V}=\tanh V,
$$

then the spacetime can be written as

$$
\mathrm{d} s^{2}=\omega^{4 / 3} \bar{\omega}^{2}\left[H(\tilde{U}, \tilde{V}) \mathrm{d} \tilde{U} \mathrm{~d} \tilde{V}+\mathrm{d} z^{2}+r^{2} \mathrm{~d} \varphi^{* 2}\right],
$$

with

$$
H=\frac{N_{1}^{2}}{N_{2}^{2}} \frac{1}{\kappa^{2} \operatorname{arctanh} \tilde{U} \operatorname{arctanh} \tilde{V}\left(1-\tilde{U}^{2}\right)\left(1-\tilde{V}^{2}\right)} .
$$

We can write $r$ and $t$ as

$$
r=r_{H}+(\operatorname{arctanh} \tilde{U} \operatorname{arctanh} \tilde{V})^{\frac{1}{2 \kappa \alpha}}, \quad t=t_{H}+\left(\frac{\operatorname{arctanh} \tilde{V}}{\operatorname{arctanh} \tilde{U}}\right)^{\frac{1}{2 \kappa \beta}},
$$

with

$$
\alpha=\frac{r_{H}}{4\left(r_{H}+b_{2}\right)^{3}}, \quad \beta=\frac{1}{4 C_{2}\left(t_{H}+b_{3}\right)^{3}} .
$$

Observe that $N_{1}$ and $N_{2}$ can be expressed in $(\tilde{U}, \tilde{V})$. The Penrose diagram is drawn in Figure 4(b). Note that $\mathrm{d} s^{2}$ and $H$ are invariant under $\tilde{U} \rightarrow-\tilde{U}$ and $\tilde{U} \rightarrow-\tilde{U} \cdot \tilde{g}_{\mu \nu}$ is regular everywhere and conformally flat. The "scale-term" $\mathrm{H}$ is consistent with the features of the Penrose diagram. Now we have still the $\varphi$ dependency. We assume no z-dependency. It is expected that the differential equation for $\omega$ can be separated in a $(U, V)$ part and a $\varphi$
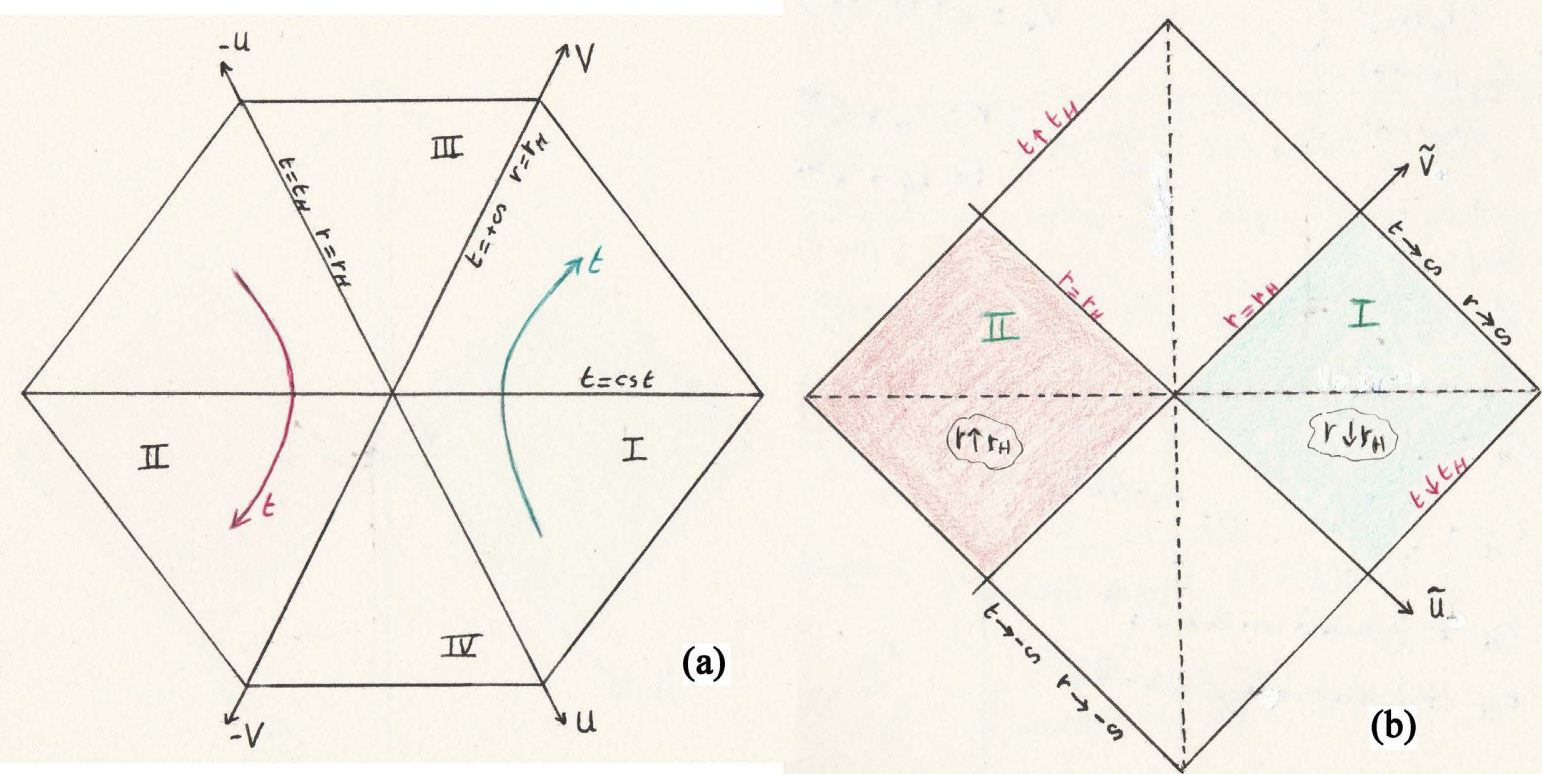

Figure 4. Plot of the Kruskal diagram for $\bar{g}_{\mu v}$ in $(U, V)$ coordinates (a). The antipodal map between region I and II is quite clear here. If one approaches the horizon from the outside and passes the horizon, one approaches from the "other side" the horizon. One can also plot the Kruskal diagram for $\bar{g}_{\mu v}$ in $(\tilde{U}, \tilde{V})$ coordinates (b). 
part. The method of 't Hooft can then be applied. In the next sections, we will briefly come back to this issue.

\section{Related Issues of the New Black Hole Solution}

\subsection{Treatment of the Quantum Fields}

The physical identification in the de Sitter spacetime of $P(X)$ and $\hat{P}(\hat{X})$ are considered as different representations in Kruskal space of one and the same Schwarzschild event. There is only one world with one singularity and one exterior region. Fields which are symmetric under Jare identified as

$$
\Psi_{J S}=\frac{1}{2}[\Psi(X)+\hat{\Psi}(\hat{X})] .
$$

One then builds these fields from fields with arguments specified in [9]. Each of these fields, positive or negative frequency in I, can be extended to global spacetime surfaces. However, due to the time reversal, the inner product on the full Hilbert space has zero norm for the symmetric fields. One then defines negative frequency functions $\Psi_{(-)}^{\uparrow}(X)=\Psi_{(+)}^{\downarrow}(J X)$ and $\Psi_{(-)}^{\downarrow}(X)=\Psi_{(+)}^{\uparrow}(J X)$, where the arrows stands for the solutions on the future/past singularity. The symmetric (anti-) solutions $(\varepsilon= \pm 1)$ are then

$$
\Psi_{J S}^{(1)}(X)=\frac{1}{2}\left[\Psi_{(+)}^{\uparrow}(X)+\varepsilon \Psi_{(-)}^{\downarrow}(X)\right], \quad \Psi_{J S}^{(2)}(X)=\frac{1}{2}\left[\Psi_{(+)}^{\downarrow}(X)+\varepsilon \Psi_{(-)}^{\uparrow}(X)\right] .(39)
$$

Introducing then reflection and transmission coefficients, one can construct a wave function regular at the singularities, $\Psi_{J S}^{(r)}=\frac{\varepsilon K}{(K+\varepsilon)^{2}}\left[\Psi_{J S}^{(1)}+\varepsilon \Psi_{J S}^{(2)}\right]$, with $K=\mathrm{e}^{\pi \omega / \kappa}, \kappa=1 / 4 M$. Thereafter, one constructs hermitian field operators for the Fock space. Next, one needs the renormalized expectation value of the stress-energy tensor $\left\langle T_{\mu \nu}\right\rangle$ in the "semiclassical" equations of Einstein $G_{\mu \nu}=8 \pi G\left\langle T_{\mu \nu}\right\rangle$. If one assumes that there is a $r=0$ singularity, then back-reaction will be small in the vicinity of the horizon (at least for massless fields). The spacetime can then be approximated by Schwarzschild geometry. The mass will decrease slowly with time and evaporates. In a flat spacetime, this is easily done, because the vacuum is well defined. One can calculate the zero-energy state and can construct finite quantum operators. In curved spacetime, the vacuum state is dependent of the boundary condition for the propagators (positive frequency modes). In principle, we can follow the method of Sanchez (for the de Sitter spacetime) for the dilaton field and our "un-physical" spacetime $\bar{g}_{\mu v}(\Lambda=0)$,

$$
\left\langle\bar{\omega}^{2}\right\rangle \bar{G}_{\mu \nu}=\left\langle T_{\mu \nu}^{(\omega)}\left(\bar{\omega}, \bar{g}_{\mu \nu}\right)\right\rangle-\left\langle\bar{\omega}^{2}\right\rangle \mathcal{E}_{\mu \nu},
$$

where $T_{\mu \nu}^{(\omega)}$ depends on the geometry and boundary conditions (see Equation (19)). Further, $\left\langle T^{(\omega)}\right\rangle=-\left\langle\bar{\omega}^{2}\right\rangle \bar{R}$, because $\mathcal{E}_{\mu \nu}$ is traceless. We have now contributions from the antipode:

$$
\left\langle T_{\mu \nu}^{(\omega)}\right\rangle \rightarrow\left\langle T_{\mu \nu}^{(\omega)}\right\rangle \pm\left\langle\hat{T}_{\mu \nu}^{(\omega)}\right\rangle, \quad\left\langle\bar{\omega}^{2}\right\rangle \rightarrow\left\langle\bar{\omega}^{2}\right\rangle \pm\left\langle\widehat{\bar{\omega}}^{2}\right\rangle .
$$

In the simplified de Sitter space, one then easily construct Green functions [10] 


$$
\begin{aligned}
& G_{\alpha J S}\left(X, X^{\prime}\right)=\mathrm{e}^{2 \alpha}\left[G\left(X, X^{\prime}\right)+G\left(X, J X^{\prime}\right)\right], \\
& G_{\alpha J A}\left(X, X^{\prime}\right)=\mathrm{e}^{2 \alpha}\left[G\left(X, X^{\prime}\right)-G\left(X, J X^{\prime}\right)\right],
\end{aligned}
$$

with $\alpha$ labels the one parameter family of the de Sitter vacua. The expectation values for a scalar field and the energy momentum tensor can then be calculated. One obtain, for example [9],

$$
\left\langle\widehat{\Phi_{J S, J A}^{2}}\right\rangle=\frac{1}{16 \pi \cos \pi v}\left[m^{2}+\left(\xi-\frac{1}{6}\right) R\right],
$$

with $v=\left(9 / 4-M^{2} / H^{2}\right)^{1 / 2}, M^{2}=m^{2}+\xi R, \mathrm{~m}$ the mass of the field and $H \sim \Lambda$. Recently, a different analysis of perturbative quantum gravity on the de Sitter spacetime was done by Sofi, et al. [33].

In our case we have no scalar field, but instead $\omega$. The expression for $T_{\tilde{U} \tilde{U}}$ becomes [34]

$$
T_{U U}^{(\omega)}=\frac{c_{1} \mathrm{e}^{-2 c_{1} U}}{c_{3}^{2} c_{4}^{2}\left(c_{2} \rho+c_{3}\right)^{2}}\left(c_{2} c_{3} \rho^{2} F(U)-c_{1}^{2} c_{2}^{2} \rho^{2}+c_{1} c_{3}^{2}\right),
$$

which can be used to evaluate the expectation value. In order to apply the full antipodal map, one includes the $\varphi$-dependency in the dilaton equation. The relevant operator (d'Alembertian) can be separated in the used coordinate system. The relevant $\varphi$ contribution comes from periodic Mathieu functions (in variable $\varphi$ ). They converge uniformly on all compact sets in the $z$-plane. Next, one applies the method of 't Hooft, by expanding the position variables $u^{ \pm}(z, \varphi)$ and momentum distributions $p^{ \pm}(z, \varphi)$ in the partial waves of Mathieu functions $s^{6}$. Further, one then calculates the gravitational shift $\delta \tilde{U}(z, \varphi)$, in order to carry a particle over from I to II, or back [7], using the Shapiro delay.

\subsection{The Surface Gravity and the Conformal Gauge}

Since we have now the description of the antipodal map in our black hole spacetime, we will look more closely at the conformal invariance. First of all, one should rely in the dynamical situation on (conformal) Killing vectors in order to describe the spacetime symmetries. Our Lagrangian is conformal invariant under Equation (14), so we can use the freedom of the conformal factor $\Omega$. Remember, different $\omega$ means different notion of the vacuum state for the in-going and outside observer, so they will use different conformal gauge freedom. It is desirable that for the out-going observer, the surface gravity of the horizon is conformal invariant. Further, conformal transformations must preserve affinely parameterized null geodesics. This will deliver $\Omega$ for the in-going observer. We can define out-going and in-going null normals [20] for $\bar{g}_{\mu v}$

$$
\begin{aligned}
& \bar{l}^{\mu}=\left(1, N \sqrt{N^{2}-r^{2} N^{\varphi 2}}, 0,0\right), \\
& \bar{m}^{\mu}=\left(-\frac{1}{2 r^{2} N^{\varphi 2}-2 N^{2}},-\frac{N}{2 \sqrt{N^{2}-r^{2} N^{\varphi 2}}}, 0,0\right) .
\end{aligned}
$$

${ }^{6}$ So the spherical harmonics are replaced by the Mathieu harmonics. 
with $\bar{l}^{\mu} \bar{l}_{\mu}=\bar{m}^{\mu} \bar{m}_{\mu}=0, \bar{l}^{\mu} \bar{m}_{\mu}=-1$. The surface gravity then becomes

$$
\kappa=2 N\left(\partial_{r}\left(\sqrt{N^{2}-r^{2} N^{\varphi 2}}\right)+\partial_{t}\left(\frac{1}{N}\right)\right)=2 N\left(\partial_{t} \sqrt{\bar{g}_{r r}}-\partial_{r} \sqrt{\bar{g}_{t t}}\right) .
$$

This is consistent with the metric definition of $\kappa$.

\subsection{The Meaning of the Warped Spacetime}

Let us now return to $g_{\mu v}=\omega^{4 / 3} \bar{\omega}^{2} \bar{g}_{\mu v}$. In the CDG setting, the evaporation of the black hole is also determined by the complementarity transformation of $\omega$ between the in-going and outside observer. Our spacetime is now $\left(b_{4}=b_{3} b_{2}\right)$

$$
\begin{aligned}
d s^{2}= & \omega^{4 / 3} \bar{\omega}^{2}\left[\left(C_{2}\left(t+b_{3}\right)^{4}+C_{3}\right) \frac{10 b_{2}^{3} r^{2}+20 b_{2}^{2} r^{3}+15 b_{2} r^{4}+4 r^{5}+C_{1}}{5 r^{2}}\right. \\
& \left.\cdot\left(-\mathrm{d} t^{* 2}+\mathrm{d} r^{* 2}\right)+\mathrm{d} z^{2}+r^{2}\left(\mathrm{~d} \varphi+\frac{N^{\varphi}}{N_{2}^{2}} \mathrm{~d} t^{*}\right)^{2}\right],
\end{aligned}
$$

with

$$
\omega^{4 / 3} \bar{\omega}^{2}=\frac{1}{\left(r+c_{2}\right)^{2}\left(t+c_{3}\right)^{2}\left(r+b_{2}\right)^{2}\left(t+b_{3}\right)^{2}},
$$

We observe that $\omega^{4 / 3} \bar{\omega}^{2}$ approaches zero for coordinate time $t \rightarrow \infty$, so $g_{\mu v}$ shrinks to zero, so the distant observer sees a gradually shrinking black hole when the metric time runs to infinity. Further, the only contribution from the $5 \mathrm{D}$ spacetime is the $\omega^{4 / 3}$. Remarkable, the projected Weyl component is necessary in order to obtain the same form of $N^{2}$ and to avoid naked singularities. So $\omega^{4 / 3}=\left[\left(r+c_{2}\right)^{2}\left(t+c_{3}\right)^{2}\right]^{-1}$ is the "scale" term from the $5 \mathrm{D}$ warped spacetime (the warpfactor in the RS model is the product of $y$-dependent part and $\omega$ part). Suppose one wants combine the conformal transformation with an internal symmetry transformation, i.e., a spacetime transformation. In particular, the scale transformations. One can proof in that case, $\square \log \Omega=0$ [21], which is consistent with our 2D null hypersurface of Equation (32). Further, in dimension $n \neq 4$ only the scale-invariant theories based upon scalar fields (so $\omega$ from 5D) are conformally invariant. Conclusion: $\bar{\omega}$ of our $\bar{g}_{\mu v}$ can be used in non-vacuum models. An additional advantage of the warped spacetime in connection with cosmology and hierarchy problem was already mentioned in the introduction. A new aspect will be the embedding of the $5 \mathrm{D}$ in the $4 \mathrm{D}$ spacetime and the relation with the $3 \mathrm{D}$ BTZ blackhole solution.

\subsection{The Relation with the 3D Baňados-Teitelboim-Zanelli Black Hole}

In the spacetime under consideration, the $\mathrm{d} z^{2}$ term can be omitted. One obtains then the 3D Baňados-Teitelboim-Zanelli (BTZ) black hole spacetime. It solves the Einstein equations with a negative cosmological constant [35]. The BTZ solution is related to the AdS/CFT correspondence and intensively studies in connection with black hole entropy issues. However, we should like to take 
the cosmological constant zero. In a former study [34], an exact solution was found in a CDG setting in Eddington-Finkelstein retarded coordinates $(U, \rho)$ (or advanced $V$ ) where the antipodal map $(U, V, \varphi) \rightarrow(-U,-V, \varphi+\pi)$ is applicable:

$\mathrm{d} s^{2}=\frac{\mathrm{e}^{-2 c_{1} U}}{\left(c_{2} \rho+c_{3}\right)^{2}}\left[ \pm \frac{c_{1}\left(c_{3}^{2}-c_{2}^{2} \rho^{2}\right)}{c_{2} c_{3}} \mathrm{~d} U^{2}-2 \mathrm{~d} U \mathrm{~d} \rho+\mathrm{d} z^{2}+\rho^{2}(\mathrm{~d} \xi+F(U) \mathrm{d} U)^{2}\right]$

which is Ricci flat, while $\tilde{R}^{(4)}=\frac{6 c_{1} c_{2}}{c_{3}}$. The function $F(U)$ will be fixed when matter terms are incorporated (i.e. for example, a scalar gauge field). The metric Equation (49) will then contain a term $b(U, \rho)^{2} \mathrm{~d} \varphi^{2}$ and a relation like $\left(N^{\xi}\right)^{\prime}=\frac{b}{\eta^{2} X^{2}+\omega^{2}}$ will be obtained. It has no curvature singularity. The location of the apparent horizon in $U$ :

$$
\rho_{A H}= \pm \frac{c_{3}}{\sqrt{c_{2}\left(c_{2}+\frac{c_{3}}{c_{1}} F(U)^{2}\right)}}
$$

with

$$
\frac{\mathrm{d} \rho}{\mathrm{d} U}=\frac{1}{2} \mathrm{e}^{-2 c_{1} U} \cdot \begin{cases}-\frac{c_{1}}{c_{2} c_{3}} & \rho \rightarrow 0 \\ \frac{c_{3} F(U)^{2}+c_{1} c_{2}}{c_{2}^{2} c_{3}} & \rho \rightarrow \infty \\ 0 & \rho=\rho_{A H}\end{cases}
$$

which is independent of $\omega$. Here $C_{i}$ are constants and $F(U)$ a function determined by the non-diagonal contribution. Further, we have

$$
\lim _{\rho \rightarrow 0} g_{U U} \rightarrow \pm \frac{c_{1}}{c_{2} c_{3} \mathrm{e}^{2 c_{1} U}}
$$

So when the evaporation speeds up, it approaches zero. We are dealing here with null-radiation in the $(\rho, z)$-plane. One could compare this solution with that found by Chan [36] in standard GR of a spinning black hole. They also find a solution for $F(U)$ which is determined by an energy-momentum tensor of null spinning dust. It is again curious that the "uplifted" BTZ has a solution, comparable with the "up-lifted" 5D solution.

\section{Metric Fluctuation and Hawking Radiation}

In the original deviation of the Hawking radiation, one uses the propagation of a linear quantized field in a classical background metric. However, near the horizon, high-frequencies metric fluctuations can contribute to the vacuum polarization and the impact of gravitational back reactions can be large. These zero-point fluctuations result in a modification of the Hawking radiation by gravitational waves [37]. One could question what the effect is of these waves in our 
CDG model, where we have instead the dilaton field. Of course, one should need a quantum gravitational approach, which is not available yet. So need some approximation. However, effect of the scattering of these quanta at the horizon can be investigated in the context of the antipodal mapping considered here. ${ }^{7}$ Without the contribution of the metric fluctuations, the mean number of quanta reaching $\mathcal{J}^{+}$takes the form

$$
\left\langle\bar{n}_{\lambda}\right\rangle_{0} \sim \frac{1}{\mathrm{e}^{2 \pi E_{+} / \kappa}-1},
$$

with $E_{+}$the energy measured at $\mathcal{J}^{+}$for the out modes. This is the Planck distribution with temperature $T=\kappa / 2 \pi=(8 \pi M)^{-1}$. The correction terms can then be calculated by using the s-modes of a quantum massless scalar field and by using the fact that the in-going and out-going modes decouple [37]. One makes use of the mean energy flux, by calculating $\mathrm{d} E / \mathrm{d} U=4 \pi r^{2}\left\langle T_{U U}\right\rangle_{\text {ren }}$, where the renormalized surface gravity is used. However, in this approximation, the reflection conditions are at $r=0$, with in our antipodal map must be revised (we have no inside). We can use the $(U, U)$ energy-momentum component of our model and can apply Equation (41) for the antipodal contribution.

Notice that the meaning of the local dilaton $\bar{\omega}$, is twofold. First, it determines the metric fluctuations (one also must incorporate in the dilaton equation the $\varphi$-dependency). Secondly, the in-going observer will use a different conformal gauge freedom $\Omega$ on $\bar{\omega}$ to describe the vacuum. Further, $\bar{\omega}$ is locally unobservable, unless we include metric fluctuations (gravitational waves. It will be necessary to compare this with the usual contribution using the BunchDavies method (and to taken into count the antipodal contribution). Note that the outside observer will use a different gauge and he/she experiences a mass $\sim \omega^{2} N^{2}$ and Hawking radiation $\sim \partial_{U}\left(\omega^{2} N^{2}\right)$, while for the in-going observer it is part of his vacuum. On the other hand, the outside observer is not aware of the antipodal identification. One could also say that they disagree about the observed scales. Or differently stated, they disagree about the back reaction from the Hawking radiation.

\section{Conclusions}

We investigated the conformal dilaton gravity model on a warped 5D spacetime, where the warp factor is interpreted as a dilaton field, to be treated as a renormalized quantum field. This approach is very suitable when one is dealing with a high curvature situation, for example, in the vicinity of the horizon of a black hole spacetime. It is a promising route to tackle the problems arising in quantum gravity models, such as the loss of unitarity when one investigates the Hawking radiation, emitted during the final stage of a black hole. Moreover, it could solve the information and firewall paradox. The basic concept behind the model is

${ }^{7} \mathrm{~A}$ suitable approximation is the high-frequency approximation applied to a Vaidya spacetime, where the not-flat background spacetime is distorted by the gravitational waves [38]. A recent application was provided by Slagter [39] [40]. 
conformal invariance, spontaneously broken when matter fields are incorporated in the Einstein-Hilbert action. The conformal symmetry group contains the antipodal map, so it is quite natural to apply the antipodal map on the black hole spacetime. It then turns out that the notion of the interior of the black hole changes dramatically, i.e., there is no inside.

In this manuscript, we find an exact time dependent solution in the conformal dilaton gravity model on a warped $5 \mathrm{D}$ spacetime. The spacetime is written as ${ }^{(5)} g_{\mu v}=\omega^{4 / 3(5)} \tilde{g}_{\mu v}$ and ${ }^{(4)} \tilde{g}_{\mu v}=\bar{\omega}^{2(4)} \bar{g}_{\mu v}$. In our model, $\omega$ can be seen as the contribution from the bulk, while $\bar{\omega}$ is the brane component. It is conjectured that the different conformal gauge freedom, $\Omega$, the in-going and outside observers possess, can be calculated by demanding a conformal invariant surface gravity and the preservation of affinely parameterized null geodesics. This means that the complementarity is expressed by the different notion of the vacuum state. The solution guarantees regularity of the action when $\omega \rightarrow 0$. We don't need a Weyl term in the action (generates negative metric states). Instead, we have a contribution from the bulk, i.e., the electric part of the 5D Weyl tensor. It is remarkable that the $5 \mathrm{D}$ field equations and the effective $4 \mathrm{D}$ equations can be written for general dimension $n$, with $n=4,5$. The energy-momentum tensor of the time-dependent dilaton, determining also the Hawking radiation, can be calculated exactly. By suitable choice of the parameters, the spacetime $\bar{g}_{\mu v}$ can be regular and singular free. In context of quantization procedures, counter terms in an effective action will cause problems, only in the bulk spacetime of the "large" extra dimension and not for the brane spacetime. When the extra dimensional volume is significantly above the Planck scale, then the true fundamental scale can be much less than the effective scale $10^{19} \mathrm{GeV}$. This means that no UV cut-off is necessary on the brane. This exact solution, nonetheless without mass terms, can be used to tackle the deep-seated problem of the black hole complementarity: the infalling and outside observer experience different $\omega$ by the choice of $\Omega$. The solution fits also very well in the antipodal mapping, when crossing the horizon. The Penrose diagram for $\bar{g}_{\mu v}$, in suitable Kruskal coordinates, shows the features of the antipodal map of region I on region II: the inside of the black hole is removed. The in-going observer, when crossing the horizon, turns up at "the other side" of the horizon. The next task is to incorporate mass into our model and investigate the dilaton-scalar field interaction. The conformal invariance will then spontaneously be broken.

\section{Acknowledgements}

This research was supported by Asfyon, Astronomisch Fysisch Onderzoek Nederland.

\section{Conflicts of Interest}

The author declares no conflicts of interest regarding the publication of this paper. 


\section{References}

[1] Hawking, S. (1974) Nature, 248, 30-31. https://doi.org/10.1038/248030a0

[2] Hawking, S. (1975) Communications in Mathematical Physics, 43, 199-220. https://doi.org/10.1007/BF02345020

[3] 't Hooft, G. (1993) Dimensional Reduction in Quantum Gravity. Conference on Highlights of Particle and Condensed Matter Physics (SALAMFEST), Trieste, 8-12 March 1993, 184.

[4] Page, D.N. (1993) Physical Review Letters, 71, 3743-3746. https://doi.org/10.1103/PhysRevLett.71.3743

[5] Almheiri, A., Marolf, D., Polchinski, J. and Sully, J. (2013) JHEP, 2, 62. https://doi.org/10.1007/JHEP02(2013)062

[6] 't Hooft, G. (2016) Foundations of Physics, 46, 1185-1198. https://doi.org/10.1007/s10701-016-0014-y

[7] 't Hooft, G. (2018) The Firewall Transformation for Black Holes and Some of Its Implications.

[8] 't Hooft, G. (1984) Journal of Geometry and Physics, 1, 45-52. https://doi.org/10.1016/0393-0440(84)90013-5

[9] Sanchez, N. and Whiting, B.F. (1987) Nuclear Physics B, 283, 605-623. https://doi.org/10.1016/0550-3213(87)90289-6

[10] Sanchez, N. (1986) Two- and Four-Dimensional Semi-Classical Gravity and Conformal Mappings.

[11] Folacci, A. and Sanchez, N. (1987) Quantum Field Theory and the Antipodal Identification of de Sitter Space. Elliptic Inflation.

[12] Schrödinger, E. (1957) Expanding Universe. Cambridge Univ. Press, Cambridge.

[13] 't Hooft, G. (2018) Discreteness of Black Hole Microstates.

[14] 't Hooft, G. (2018) Foundations of Physics, 48, 1134-1149. https://doi.org/10.1007/s10701-017-0133-0

[15] 't Hooft, G. (2015) Diagonalizing the Black Hole Information Retrieval Process.

[16] Zakharov, V.D. (1973) Gravitational Waves in Einstein's Theory. John Wiley \& Sons, Inc., New York.

[17] Wald, R.M. (1994) Quantum Field Theory in Curved Spacetime and Black Hole Thermodynamics. The Univ. of Chicago Press, Chicago.

[18] Codello, A., D’Odorico, G., Pagani, G. and Percacci, R. (2013) Classical and Quantum Gravity, 30, Article ID: 115015. https://doi.org/10.1088/0264-9381/30/11/115015

[19] Alvarez, E., Herrero-Valea, M. and Martin, C.P. (2014) JHEP, 10, 214. https://doi.org/10.1007/JHEP10(2014)115

[20] Slagter, R.J. (2021) Conformal Dilaton Gravity and Warped Spacetimes in 5D.

[21] Felsager, B. (1998) Geometry, Particles and Fields. Springer, New York. https://doi.org/10.1007/978-1-4612-0631-6

[22] Mannheim, P.D. (2005) Progress in Particle and Nuclear Physics, 56, 340-445. https://doi.org/10.1016/j.ppnp.2005.08.001

[23] Randall, L. and Sundrum, R. (1999) Physical Review Letters, 83, 3370-3373. https://doi.org/10.1103/PhysRevLett.83.3370

[24] Randall, L. and Sundrum, R. (1999) Physical Review Letters, 83, 4690-4693. 
https://doi.org/10.1103/PhysRevLett.83.4690

[25] Slagter, R.J. and Pan, S. (2016) Foundations of Physics, 46, 1075. https://doi.org/10.1007/s10701-016-0002-2

[26] Maldacena, J. and Milekhin, A. (2021) Physical Review D, 103, Article ID: 066007. https://doi.org/10.1103/PhysRevD.103.066007

[27] Maldacena, J. (2011) Einstein Gravity from Conformal Gravity.

[28] Islam, J.N. (1985) Rotating Fields in General Relativity. Cambridge Univ. Press, Cambridge. https://doi.org/10.1017/CBO9780511735738

[29] Shiromizu, T., Maeda, K. and Sasaki, M. (2000) Physical Review D, 62, Article ID: 024012. https://doi.org/10.1103/PhysRevD.62.024012

[30] Shiromizu, T., Maeda, K. and Sasaki, M. (2003) Physical Review D, 7, Article ID: 084022. https://doi.org/10.1103/PhysRevD.67.084022

[31] Maartens, R. and Koyama, K. (2010) Living Reviews in Relativity, 13, 5. https://doi.org/10.12942/lrr-2010-5

[32] Strauss, N.A., Whiting, B.F. and Franzen, A.T. (2020) Classical Tools for Antipodal Identification in Reissner-Nordstrom Spacetime. https://doi.org/10.1088/1361-6382/ab9a9d

[33] Sofi, A.H., Akhoon, S.A., Rather, A.A. and Maini, A. (2015) Open Access Library Journal, 2, 1. https://doi.org/10.1088/1361-6382/ab9a9d

[34] Slagter, R.J. (2019) On the Dynamical BTZ Black Hole in Conformal Gravity. In Spacetime 1909-2019. Proceedings Second H. Minkowski Meeting, Albena, May 2019, 17-37.

[35] Compère, G. (2019) Advanced Lectures on General Relativity. Lecture Notes in Physics 952, Springer, Heidelberg. https://doi.org/10.1007/978-3-030-04260-8

[36] Chan, J.S.F., Chan, K.C.H. and Mann, R.B. (1996) Physical Review D, 54, 1535-1539. https://doi.org/10.1103/PhysRevD.54.1535

[37] Barrabès, C. and Hogan, P.A. (2013) Advanced General Relativity. Oxford Univ. Press, Oxford. https://doi.org/10.1093/acprof:oso/9780199680696.001.0001

[38] Choquet-Bruhat, Y. and Geroch, R.P. (1969) Communications in Mathematical Physics, 14, 329-335. https://doi.org/10.1007/BF01645389

[39] Slagter, R.J. and Miedema, P.G. (2020) Monthly Notices of the Royal Astronomical Society, 459, 3054. https://doi.org/10.1093/mnras/staa3840

[40] Slagter, R.J. (2021) New Evidence of the Azimuthal Alignment of Quasars Spin Vector in the LQG U1.28, U1.27, U1.11, Cosmologically Explained. https://doi.org/10.20944/preprints202103.0560.v1

[41] Toth, G. (2002) Finite Möbius Groups, Minimal Immersions of Spheres and Moduli. Springer, Heidelberg. https://doi.org/10.1007/978-1-4613-0061-8

[42] Klein, F. (1888) Lectures on the Icosahedron and the Solution of Equations of the Fifth Degree. Trübner \& Co, London. 


\section{Appendix: The Quintic Horizon Equation and Related Issues}

Our quintic polynomial, determining the horizons,

$$
f=r^{5}+\frac{15}{4} b_{2} r^{4}+5 b_{2}^{2} r^{3}+\frac{5}{2} b_{2}^{3} r^{2}+\frac{C_{1}}{4}=0,
$$

can be written by a, so-called Tschirnhaus transformation, in the form

$$
r^{5}-\frac{15 b_{2}}{16}\left(C_{1}+b_{2}^{5}\right) r^{2}-\frac{125 b_{2}^{3}}{256}\left(C_{1}+b_{2}^{5}\right) r-\frac{1}{16}\left(C_{1}+b_{2}^{5}\right)^{2}=0
$$

By scaling, this form can be reduced to the Bring-Jerrard form $r^{5}+r-c$, with $c$ a function of $b_{2}$ and $C_{1}$ [41]. There is an interesting relation between the symmetry group of the icosahedron and our quintic equation. The symmetry group is isomorphic with the Galois group $A_{5}$ (of an irreducible quintic polynomial). The icosahedron is dual to the dodecahedron, i.e., their symmetries are isomorphic. The $A_{5}$ is interesting in physics, because it is a simple group having no invariant subgroups. It has three orbits, which are invariant under the antipodal map. So the connection with the Möbius group is clear (see section 2.3). For details, we refer to Toth [41]. It was Klein [42], who first became aware of the relation between the solutions of the quintic equation and the icosahedron.

It is conjectured that our quintic polynomial (Equation (54)) has a deep-seated relation with the $5 \mathrm{D}$ spacetime solution. Further, it is remarkable that the resulting quintic equation is independent of the dimension of our manifold $(n=4,5)$. Moreover, the nice fitting of the antipodal map in our model cannot be a coincident. From Equation (26) we observe that the derivative of $f$ is $5 r\left(r+b_{2}\right)^{3}$. So it is expected that our quintic equation results from a immersion ${ }^{8}$ of a closed surface $S$ in $\mathbb{R}^{3}$ into $\mathbb{R}^{4}$.

This is currently under investigation by the author.

\footnotetext{
${ }^{8} \mathrm{An}$ immersion is a differentiable function between differentiable manifold whose derivative is everywhere injective. It is also a topological embedding.
} 